- Identify patients who need holistic symptom assessment.

- Contribute to future planning and complex decision-making.

- Sign-post to support that is local to the patient.

- Advocate for improved person-centred care during hospital admission.

- Facilitate continuity of care during transition from children's services.

Methods and impact The cross-service multidisciplinary team meet bi-monthly. Data for all outputs and activity are recorded. Palliative care outputs of the meeting include plans for advance care discussions; symptom management advice; discussion with local community palliative teams; and referral to a bespoke young adult palliative care clinic. Additional benefits include peer support within the group and teaching opportunities with specialty teams. Further time is needed to evaluate the impact on patient experience although initial feedback from patients and professionals has been positive.

Conclusions We describe a new model of palliative care input for a patient group who are known to have multiple unmet needs. Outcomes to date demonstrate that the model is sustainable, supportive to staff, harnesses and enhances generalist palliative care skills, and is likely to improve patient experience and continuity of care.

\section{P-23 HOSPICE HEARTY MEALS: TACKLING FOOD INEQUALITY DURING THE PANDEMIC}

Donna Bruce, Margaret Wilkie. St Andrew's Hospice, Lanarkshire, UK

\subsection{6/spcare-2021-Hospice.44}

Hospice Hearty Meals was inspired by our patients in response to COVID-19. Due to reduced activity, some patients' function decreased, affecting their ability to cook nutritious meals. Others, who relied on family/friends to prepare meals, were impacted by shielding.

St Andrew's Hospice suspended outpatient services to comply with Government guidance and reduce the risk to vulnerable patients/carers. During telephone and video support calls a need was identified in accessing nutritious meals. Clients were no longer participating in regular exercise/activity and were losing function/dexterity and struggled to prepare meals, they became reliant on ready meals/snacks. Others depended on family/friends to support them with home-cooked meals and this wasn't an option. Carers advised the catering burden on them during the pandemic and lack of respite meant they were struggling and cooking less. Carers were fearful of visiting shops and having no-one to stay with their loved one.

Method Costings were included for a 12 week trial, for the most vulnerable patients/carers. A fresh, nutritious, two-course meal (soup/main or main/pudding) was prepared onsite and packaged based on requirements (for oven reheating or microwave). Delivered three times per week.

Results and conclusions 579 meals were delivered in the 12 weeks of the project. $100 \%$ of respondents rated the service as excellent. Feedback found this service to be invaluable during uncertain times. Patients/carers reported that they ate well, were less anxious and felt more able to cope.

'Great service especially when going through such a hard time.'

'This service has improved my health greatly by relieving some of the stresses of lockdown.'
'Excellent service which saved 'valuable time' to spend together[with loved one] before death.'

Moving forward Due to this success we applied for funding which allowed us to continue and to date we have provided over 1000 meals to patients/carers across Lanarkshire.

\section{Mental health, wellbeing, bereavement and communication}

\section{P-24 WORKING IN BEREAVEMENT WITH PEOPLE WITH AUTISTIC SPECTRUM CONDITION}

Andrew Schwab, Sarah Popplestone-Helm. St Richard's Hospice, Worcester, UK

\subsection{6/spcare-2021-Hospice.45}

Background A principal difference between neurotypical people and those with autistic spectrum conditions is reliance on structure and sameness (Cohmer, 2014). There is evidence that people with autistic spectrum conditions lack flexibility of thinking (Ciesielski \& Harris, 1997). The ability to react to events or small changes can trigger anxiety. Little sabotages structure and sameness more than a death.

Aims Dealing with death is difficult, and for somebody with an autistic spectrum condition, it may introduce more challenges and anxieties. Routines change, people behave unusually, emotions can be all-consuming. People with autistic spectrum conditions can experience Alexithymia, the inability to differentiate between emotions (Poquérusse, Pastore, Dellantonio et al., 2018), highlighting the need for effective and immediate emotional support pre- and post- bereavement. Hospice social workers recognised the importance of providing effective and specialised support for people with autistic spectrum conditions pre- and post- bereavement.

Methods An example of this is the use of widgets to explain death, dying and bereavement to individuals with an autistic spectrum condition. Widgets are an augmentative communication system using words and pictures to enable understanding and to processing messages. They are an ideal way to refer back to a social story or schedule to consistently communicate a message. Examples of how widgets can be used are: what happens at a funeral, what is cancer etc.

Results Feedback has been good with a high level of engagement from people with autism and learning disability. This approach led to relationships developing with services and schools around the county that support people with these conditions, facilitating consistent organisational approaches.

Conclusions Objectives are to build on achievements in terms of inclusive support. The hospice now has an inclusion and diversity working group, delivering training sessions internally and externally to raise awareness and to support others to develop practice in this area.

\section{P-25 SUPPORTING CHILDREN TO PREPARE FOR THE DEATH OF A PARENT - EXPERIENCES OF AN ACUTE HOSPITAL}

Jo Poultney, Jessica Matthews. South Warwickshire Foundation Trust, Warwick, UK

10.1136/spcare-2021-Hospice.46

Background Annually across the world a substantial number of dependent children experience the death of a parent through 